\title{
The immune imbalance in the second hit of pancreatitis is independent of IL-17A
}

${ }^{a}$ Department of Surgery, Chris Hani Baragwanath Academic Hospital, Johannesburg, South Africa.

${ }^{b}$ Department of Surgery, Faculty of Health Sciences, University of the Witwatersrand, Johannesburg, South Africa.

${ }^{c}$ Department of Surgery, Steve Biko Academic Hospital and University of Pretoria, Pretoria, South Africa.

${ }^{\mathrm{d}}$ School of Physiology, Faculty of Health Sciences, University of the Witwatersrand, Johannesburg, South Africa.

* Corresponding author:

Department of Surgery, 9th Floor Medical School, 7 York Road, Johannesburg, South Africa, 2193

E-mail address: Pascaline.Fru-Fonteh@wits.ac.za (P Fonteh) 
BACKGROUND: Severe acute pancreatitis (SAP) is characterised by two distinct clinical phases. Organ dysfunction and death is initially as a result of a systemic inflammatory response syndrome (SIRS). Systemic sepsis from infected pancreatic necrosis characterises the second phase, the so called 'second hit' of acute pancreatitis (AP). An immune imbalance during the second hit is postulated to contribute to the formation of the septic complications that occur in these patients. The pro-inflammatory T-helper (Th) 17 pathway has been shown to be an initiator of early SIRS in AP, however to date its role has not been established in the second hit in AP.

METHODS: Thirty-six patients with mild $(n=16)$, moderate $(n=10)$ and severe $(n=10)$ acute pancreatitis were enrolled. Peripheral blood samples were drawn on days 7, 9,11 and 13 of illness for analysis of routine clinical markers as well as cytokine analysis. Flow cytometry and a IL-17A ELISA was performed to determine cytokine concentrations.

RESULTS: There were no significant differences between days 7, 9, 11 and 13 for either the mild/moderate or SAP groups for IL-17A (CBA assay or ELISA), IFN- $\gamma$, TNF- $\alpha$, IL-2 or IL-4. For each of the study days, the mean IL-6 and IL-10 concentrations were significantly higher in the SAP group compared to the mild/moderate group. WCC, CRP and PCT were all significantly higher in severe acute pancreatitis over the study days.

CONCLUSIONS: An immune imbalance exists in patients with SAP, however secreted IL-17A is not responsible for the second hit in AP.

Keywords: Acute pancreatitis, immunology, interleukins 


\section{Introduction:}

$\mathrm{AP}$ is an inflammatory condition of the pancreas, as a result of various aetiologies, that initiate a varied systemic inflammatory response. The incidence of AP has increased by $43 \%$ in recent years occurring in approximately 210000 patients annually in the United States of America (1-3). The predominant causes are related to lifestyle, namely excess alcohol consumption and gallstone disease. According to the World Health Organization, $75 \%$ of deaths related to non-communicable diseases occur in low to middle income countries (4), therefore lifestyle modification has a role to play in curbing the increasing incidence of AP (3). The majority of cases of AP (80-90\%) are mild (1,5) and thus self-limiting, with an associated mortality rate of less than $1 \%(2,6)$ as opposed to severe acute pancreatitis (SAP) which develops in the remainder of cases and is associated with a mortality rate of $10-40 \%(7,8)$.

Commonly, there is a bimodal pattern of organ failure in SAP. Within the first week multi-organ failure occurs as a result of the systemic inflammatory response syndrome (SIRS) (9-11). Organ failure occurring after the first week is usually as a result of septic complications (10-13) arising from infected pancreatic necrosis. This is known as the 'second hit' of SAP. Current clinical assessments of severity only stratify $60-80 \%$ of patients accurately highlighting the deficiency in our understanding of the pathophysiology of the disease. SAP is defined by the presence of organ failure but not all organ failure carries the same prognosis (14). Furthermore not all patients with SAP develop septic complications, thus predicting which patients will have a poor clinical outcome is challenging (15).

The immunology of early AP has been elucidated. Various elements of the T-helper (Th) 1 or pro-inflammatory pathway, namely Interleukin (IL)-1, IL-2, IL-6, IL-8 and Tumor Necrosis Factor alpha (TNF- $\alpha$ ) have been implicated in the severity of pancreatitis, predicting mortality and/or the development of pancreatic necrosis (16-25). Similarly, the Th2 response or anti-inflammatory pathway has been investigated through IL-4 and IL-10, and have also been shown to predict severity and/or the development of pancreatic necrosis $(18,25,26)$. A hypo-inflammatory state, driven by the compensatory anti-inflammatory response syndrome (CARS), during the second hit has been hypothesised to result in immune paresis (17). This phase is dominated by a Th2 response, with an elevated IL-10 and suppressed TNF- $\alpha$, IL-1 $\beta$ and IL-6 (27). Immune paresis and subsequent sepsis has not been investigated in SAP.

The primary function of proinflammatory T-helper 17 (Th17) cells is eradicating excess pathogens not adequately addressed by Th1 or Th2 cells (28). Secondarily Th17 cells are potent inducers of tissue inflammation (29). IL-17A, IL-17F, IL-21 and IL-22 are pro-inflammatory cytokines produced by Th17 cells (28). Significant co-expression has been demonstrated between IL-17A and IL-22 (29). IL-23 drives the differentiation and inflammatory functions of Th17 cells (30) and alone cannot differentiate 
CD4+ T cell precursors into the Th17 cell lineage (29), IL-6 and TGF $\beta$ are required for this (27). Determining levels of IL-17A are thus representative of the Th17 response.

In experimental rat models $\mathrm{Ni}$ et al. demonstrated that that IL-17A was substantially raised during the first week of induced AP (30). Furthermore, it was demonstrated that recombinant IL-17A induces acinar cell necrosis and therefore may induce pancreatic damage (30). This experimental model, however, did not measure IL-17A during the suspected immune paresis period and no evaluation of the development of secondary septic complications was made. Similarly, in humans with mild, alcohol related AP, our unit demonstrated that IL-17A levels where elevated early in the disease process (31). In this study, we hypothesized that not only does an immune imbalance occurs in SAP, but that during the second hit in SAP, the Th17 response is active. To investigate this, the relationship of the Th1/Th2 response as well as the Th17 activity during the second hit in mild, moderate and severe AP was assessed.

\section{Materials and Methods:}

Sampling: Ethical approval was obtained from the WITS Human Research Ethics Committee (M160254). Thirty six patients diagnosed with AP, according to the revised Atlanta Classification (32) were enrolled. Only patients presenting prior to day 7 since the onset of symptoms were considered for inclusion. Patients with severe comorbidities, chronic inflammatory conditions and malignancy were excluded. Although, moderately severe AP is classified as a discreet subgroup it is associated with a mortality rate of $2 \%(33)$, similar to mild AP $<1 \%(2,6)$, hence mild and moderate AP patients were combined into one group and compared to SAP patients. Six healthy participants were recruited as controls. Sample collection took place at Chris Hani Baragwanath Academic Hospital as well as Wits Donald Gordan Medical Centre between June 2016 and July 2017. Peripheral blood samples were drawn into EDTA tubes on days 7, 9, 11 and 13 from onset of illness and processed within three hours of collection. Plasma was aspirated from the rested sample and centrifuged at $2200 \mathrm{rpm}$ for $10 \mathrm{mins}$ prior to being stored at $-80^{\circ} \mathrm{C}$. To prevent damage of the biomolecules, freeze thawing was avoided and only single use sample aliquots were prepared and used for the bioassays.

Th1/Th2/Th17 profiling: The Th1/Th2/Th17 Cytometric Bead Array (CBA) assay (BD Biosciences, La Jolla, Ca. USA) was performed as per manufacturer instructions. In summary, unique antibody-coated beads were utilized to bind to cytokines that were present in the plasma samples. A specific fluorescence was associated with each bead which was detected by the flow cytometer allowing for identification of the various cytokines within the plasma sample. A standard assay specific template provided by BD Biosciences was utilized on a LSRFortessa ${ }^{\mathrm{TM}}$ flow cytometer (BD Biosciences La Jolla, Ca. USA) for the 
experiment. Acquired data in the form of flow cytometer standard (FCS) files was quantified using Flow Cytometric Analysis $\operatorname{Program}^{\mathrm{TM}}\left(\right.$ FCAP Array ${ }^{\mathrm{TM}}$ ) software (BD Biosciences La Jolla, Ca. USA). Interferon gamma (IFN- $\gamma$ ), TNF- $\alpha$, IL-2, IL-4, IL-6, IL-10 and IL-17A concentrations were all determined from standard curves.

A confirmatory ELISA for IL-17A was performed using the BioLegend Human IL-17A Max Deluxe Set as per manufacturer's instructions. Absorbance values were quantified by using a ThermoFisher Scientific Multiskan ${ }^{\mathrm{TM}}$ GO Spectrophotometer plate reader. The IL-17A concentration for each sample was determined from a standard curve.

Clinical tests: Haematological, biochemistry and point of care assessments of white cell counts (WCC), platelets, C-reactive protein (CRP), procalcitonin (PCT), albumin, lactate and base excess (BE) were performed on all samples from all study days. Computerised tomography (CT) scans were performed where the diagnosis of AP was in doubt and on patients suspected to have developed local complications that may have required further intervention. Local complications, including necrosis, were defined as per the revised Atlanta Classification (32). Sepsis in this study was defined as either a positive blood culture or retroperitoneal air associated with a collection seen on abdominal CT scans requiring drainage. Clinical management decisions were not influenced by the study protocol. Admitting clinicians were not influenced by the study's researchers.

Statistical Analysis: Fisher's exact test was used to assess the relationship between categorical clinical variables and the disease group. APACHE II and SOFA scores were compared between disease groups using the independent samples t-test. Age and length of hospital stay was compared between the disease groups using the Wilcoxon rank sum test as the assumptions for the independent samples t-test were not met.

Between-group comparisons of the continuous clinical and study variables were performed. A repeated measures mixed model with an auto regressive (AR (1)) covariance matrix, with post-hoc comparisons (Tukey Kramer adjustment for multiple comparisons) between days (within groups) and between groups (within days) were performed to allow for any missing data. A natural $\log$ transformation of the dependent variable was carried out where necessary, to meet the assumptions of the analysis technique. Where such variables contained values of zero, an amount equal to one half of the next smallest value was added to all values before performing the transformation. The results were then back-transformed to estimated means. 
Sample size detection of a medium effect size $(\mathrm{f}=0.25)$ with $80 \%$ power at the $5 \%$ significance level, assuming a 0.5 correlation between the repeated measures, required a total sample size of 82 (41 per group). The actual sample size of 36 within this study is adequate only for the detection of large effect sizes $(\mathrm{f}=0.38)$.

\section{Results:}

Th1/Th2/Th17 cytokine profile: There were no significant differences in IL-17A levels between days 7, 9, 11 and 13 for either the mild/moderate or SAP groups using CBA assay or ELISA, nor in the IFN- $\gamma$, TNF- $\alpha$, IL-2 or IL-4 using the CBA assay (Figure I). Furthermore, no significant differences were noted between the specific days for either group. No difference was noted between the IL-17A levels (both CBA assay and ELISA) in the patients and the controls. For each of the study days, the mean IL10 level was significantly higher in the SAP group compared to the mild/moderate group (Figure I). $(\mathrm{p}=0.0003,<0.0001$, 0.0001, 0.0039 for days 7-13 respectively) Furthermore, in the mild/moderate group the mean IL-10 level was significantly higher on day 7 compared with day $9(\mathrm{p}=0.0091)$, however, no difference was detected in the remaining days. The IL-10 level was significantly higher in the SAP group compared to the control group $(\mathrm{p}=0.0003)$, but not for the mild/moderate group. The mean IL-6 level was significantly higher in the SAP group compared to the mild/moderate group for all the study days $(\mathrm{p}=<0.0001$ day $7 ; p=0.0008$ day $9 ; p=0.002$ day $11 ; p=0.013$ day 13 ) (Figure I). The mean control IL-6 level was significantly lower than that of the mild/moderate and SAP groups $(\mathrm{p}=0.0001)$.

Clinical information: Thirty-six patients aged between 22 and 78 years of age with AP were enrolled into the study. Twenty-six patients had mild/moderate disease and ten patients had severe disease. Six healthy individuals were included as study controls. An overview of the scoring systems, inflammatory markers and clinical outcomes are shown in Table I.

The mean APACHE II score for patients in the SAP group (17.7 \pm 7.7$)$ was significantly higher than that of patients in the mild/moderate group $(6.7 ; \mathrm{SD}=2.9)(\mathrm{p}<0.0001)$. The effect size was large (Cohen's $\mathrm{d}=2.4)$. Similarly, the mean SOFA score for patients in the SAP group (8.2; $\mathrm{SD}=4.8)$ was significantly higher than that of patients in the mild/moderate group $(1.7 ; \mathrm{SD}=1.0)$ $(p<0.0001)$. The effect size was large (Cohen's $d=2.6)$. 

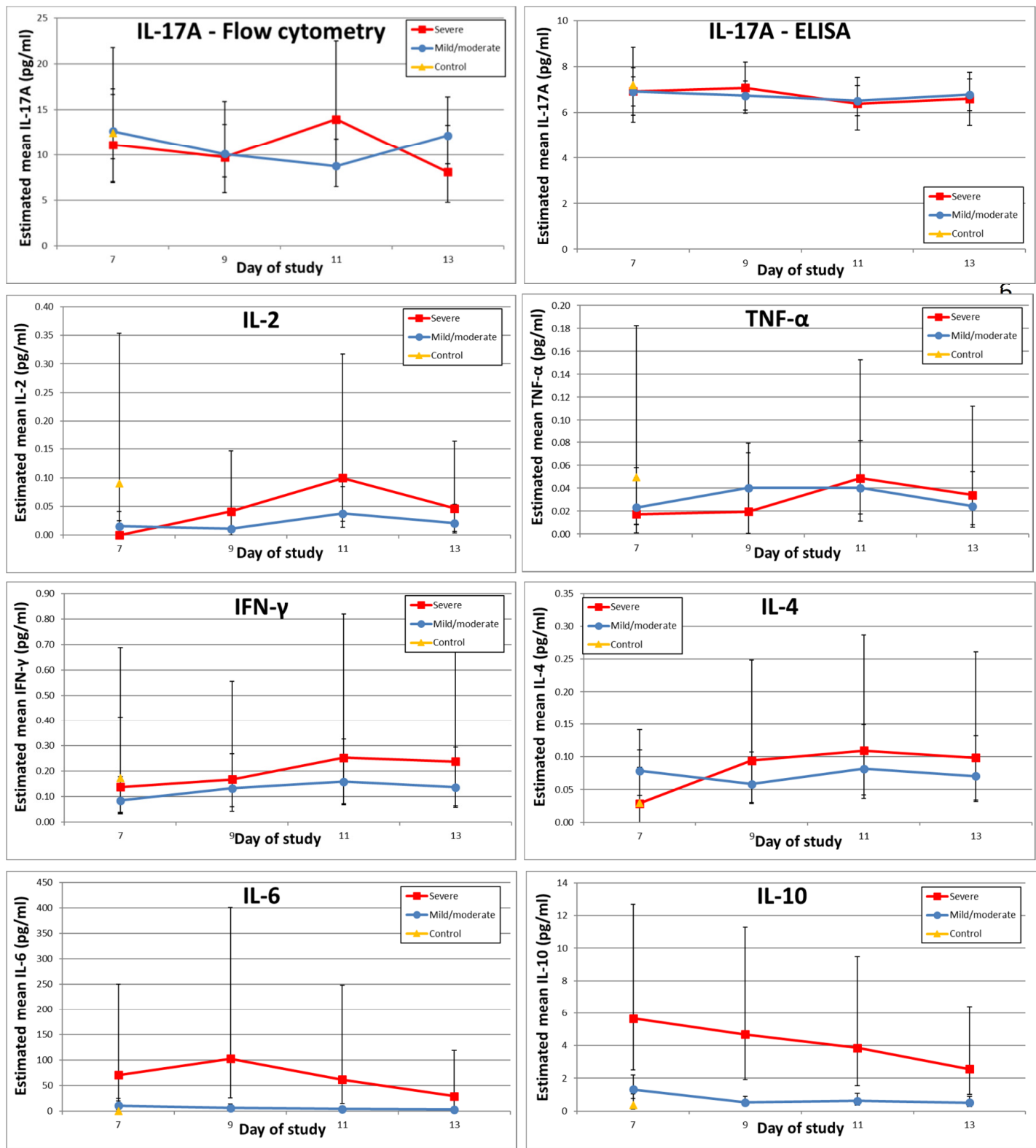

Figure I: Flow cytometry and ELISA measurements of cytokine profiles of 36 samples on days 7, 9, 11 and 13 of acute pancreatitis. The error bars denote the $95 \%$ confidence limits for the estimated means. 
Table I: Overview of scoring systems, routine inflammatory markers and clinical outcomes by severity group.

\begin{tabular}{|c|c|c|c|c|}
\hline & & Mild/moderate & Severe & p-value \\
\hline \multicolumn{2}{|c|}{ Acute pancreatitis patients (no.) } & 26 & 10 & \\
\hline \multicolumn{2}{|c|}{ Age (years) } & 45.5 & 47.7 & 0.74 \\
\hline \multirow{2}{*}{ Gender } & Male & 11 & 4 & \multirow{2}{*}{$>0.99$} \\
\hline & Female & 15 & 6 & \\
\hline \multirow{4}{*}{ Cause of Pancreatitis } & Biliary & 14 & 4 & \multirow{4}{*}{0.23} \\
\hline & Alcohol & 10 & 4 & \\
\hline & ERCP & 1 & 2 & \\
\hline & Other & 1 & & \\
\hline \multicolumn{2}{|l|}{ APACHE II score } & 6.7 & 17.7 & $<0.0001$ \\
\hline \multicolumn{2}{|l|}{ SOFA score } & 1.7 & 8.2 & $<0.0001$ \\
\hline \multirow{4}{*}{$\begin{array}{c}\mathrm{WCC}\left(\mathrm{x} 10^{9} / \mathrm{L}\right) \\
(\text { mean })^{*}\end{array}$} & Day 7 & 8.13 & 17.70 & 0.0002 \\
\hline & Day 9 & 7.95 & 19.24 & $<.0001$ \\
\hline & Day 11 & 8.41 & 18.96 & 0.0001 \\
\hline & Day 13 & 7.48 & 16.81 & 0.0001 \\
\hline \multirow{4}{*}{$\begin{array}{l}\mathrm{CRP}(\mathrm{mg} / \mathrm{L}) \\
\quad(\text { mean })^{*}\end{array}$} & Day 7 & 55.99 & 224.75 & 0.010 \\
\hline & Day 9 & 38.45 & 198.80 & 0.003 \\
\hline & Day 11 & 27.62 & 202.66 & 0.001 \\
\hline & Day 13 & 15.99 & 153.26 & 0.000 \\
\hline \multirow{4}{*}{$\begin{array}{c}\text { PCT }(\mu \mathrm{g} / \mathrm{L}) \\
(\text { mean }) *^{*}\end{array}$} & Day 7 & 0.21 & 10.00 & $<0.0001$ \\
\hline & Day 9 & 0.23 & 5.65 & $<0.0001$ \\
\hline & Day 11 & 0.16 & 3.71 & $<0.0001$ \\
\hline & Day 13 & 0.16 & 1.57 & 0.0004 \\
\hline \multirow{4}{*}{$\begin{array}{c}\text { Platelets }\left(\times 1^{9} / \mathrm{L}\right) \\
(\text { mean })^{*}\end{array}$} & Day 7 & 285.92 & 186.80 & 0.023 \\
\hline & Day 9 & 330.65 & 261.04 & 0.13 \\
\hline & Day 11 & 391.34 & 354.74 & 0.44 \\
\hline & Day 13 & 391.83 & 372.20 & 0.68 \\
\hline \multirow{4}{*}{$\begin{array}{l}\operatorname{Albumin}(\mathrm{g} / \mathrm{L}) \\
(\text { mean }) *^{*}\end{array}$} & Day 7 & 36.81 & 22.90 & $<.0001$ \\
\hline & Day 9 & 37.12 & 23.64 & $<.0001$ \\
\hline & Day 11 & 38.33 & 23.47 & $<.0001$ \\
\hline & Day 13 & 39.56 & 24.14 & $<.0001$ \\
\hline \multicolumn{2}{|l|}{ Antibiotics (\%) } & 31 & 90 & 0.0023 \\
\hline \multicolumn{2}{|l|}{ CT (\%) } & 16 & 50 & 0.020 \\
\hline \multicolumn{2}{|l|}{ Local complications (\%) } & 27 & 80 & 0.0078 \\
\hline \multicolumn{2}{|l|}{ Necrosis (\%) } & 12 & 50 & 0.024 \\
\hline \multicolumn{2}{|l|}{ Sepsis $(\%)$} & 0 & 40 & 0.026 \\
\hline \multicolumn{2}{|l|}{ Death $(\%)$} & 4 & 40 & 0.015 \\
\hline \multicolumn{2}{|c|}{ Length of Hospitalisation (days) } & 12 & 22 & 0.16 \\
\hline
\end{tabular}

* Estimated mean values from the repeated measures ANOVA 
For each of the study days, the mean WCC, CRP and PCT were significantly higher in the SAP group compared to the mild/moderate group (Table I). The CRP level in the mild/moderate group decreased significantly between days 7 and 9 , as well, as between days 11 and $13(\mathrm{p}=<0.001)$. There were no significant decreases over time in CRP in the SAP group however. The mean PCT level within the SAP group was significantly lower on day 13 compared to day $7(\mathrm{p}=0.0174)$. Only on day 7 of illness $(\mathrm{p}=0.023)$, was the mean platelet level significantly higher in the mild/moderate group compared to the SAP group. For each of the study days, the mean albumin level was significantly lower in the SAP group compared to the mild/moderate group (p $=<0.0001)$. There were no significant differences between the groups for lactate and BE values.

WCC, CRP and PCT in relation to the development of necrosis and sepsis: The mean WCC count was significantly higher in patients that developed necrosis only on day 9 , however, in patients that developed sepsis the mean WCC was significantly elevated on days 7, 9 and 13 of illness (Table II). On days 7,9 and 11 the mean CRP level was significantly higher in patients that developed necrosis compared to patients that had no necrosis, decreasing from day 7-13 both groups. Patients that developed sepsis had a significantly raised mean CRP on days 11 and 13 of illness compared to the aseptic patients (Table II). The mean PCT level was significantly higher in patients that developed necrosis and/or sepsis for all the study days (Table II) with a decreasing trend in the necrosis group from day $7-13$.

There was a significant, strong, association between use of antibiotics and disease severity $(\mathrm{p}=0.0023$; phi coefficient 0.53$)$. The proportion of patients who received antibiotics in the SAP group (90\%) was higher than in the mild/moderate group (31\%). Furthermore, a significant association between the number of CT scans and disease severity $(\mathrm{p}=0.020$; phi coefficient 0.45$)$ existed. The proportion of patients who required two or more CT scans in the SAP group (50\%) was higher than in the mild/moderate group (16\%).

There was a significant association between the development of local complications and disease severity $(\mathrm{p}=0.0078$; $\mathrm{phi}$ coefficient 0.48 ). In the SAP group $80 \%$ of patients developed local complications compared with the mild/moderate group $(27 \%)$. Furthermore, the development of necrosis was significantly associated with disease severity $(\mathrm{p}=0.024$; phi coefficient 0.41). Within the SAP group, necrosis occurred in $50 \%$ of patients as compared to $12 \%$ in the mild/moderate group. 
Table II: WCC, CRP and PCT in relation to the development of Necrosis and Sepsis.

\begin{tabular}{|c|c|c|c|c|c|c|c|}
\hline \multicolumn{8}{|c|}{ Routine Inflammatory markers in relation to the development of Necrosis and Sepsis } \\
\hline & & No necrosis & Necrosis & p-value & No sepsis & Sepsis & p-value \\
\hline \multirow{4}{*}{ WCC $\left(\times 10^{9} / L\right) *$} & Day 7 & 9.48 & 12.56 & 0.26 & 9.37 & 18.23 & 0.039 \\
\hline & Day 9 & 9.03 & 15.15 & 0.043 & 9.25 & 21.09 & 0.014 \\
\hline & Day 11 & 9.64 & 14.17 & 0.13 & 9.77 & 18.51 & 0.054 \\
\hline & Day 13 & 8.62 & 12.33 & 0.16 & 8.58 & 18.52 & 0.021 \\
\hline \multirow{4}{*}{$\operatorname{CRP}(\mathrm{mg} / \mathrm{L})^{*}$} & Day 7 & 60.68 & 240.31 & 0.027 & 71.89 & 246.38 & 0.13 \\
\hline & Day 9 & 42.66 & 199.14 & 0.015 & 52.11 & 187.14 & 0.12 \\
\hline & Day 11 & 32.96 & 163.08 & 0.012 & 38.92 & 232.21 & $\mathbf{0 . 0 3 7}$ \\
\hline & Day 13 & 22.29 & 77.20 & 0.051 & 22.90 & 239.01 & 0.0078 \\
\hline \multirow{4}{*}{ PCT $(\mu \mathrm{g} / \mathrm{L})^{*}$} & Day 7 & 0.39 & 3.16 & 0.010 & 0.37 & 33.23 & $<.0001$ \\
\hline & Day 9 & 0.36 & 2.55 & 0.019 & 0.37 & 14.24 & 0.0002 \\
\hline & Day 11 & 0.27 & 1.47 & 0.042 & 0.25 & 10.89 & 0.0002 \\
\hline & Day 13 & 0.20 & 1.16 & 0.042 & 0.18 & 20.66 & $<.0001$ \\
\hline
\end{tabular}

* LS = Least square 
Septic complications had a strong and significant association with disease severity $(\mathrm{p}=0.0036$; phi coefficient 0.57$)$. Forty percent of patients in the SAP group compared to none in the mild/moderate group developed septic complications. Death was significantly higher in the SAP group (40\%) compared to the mild/moderate group (4\%) and thus associated with disease severity $(\mathrm{p}=0.015$; phi coefficient 0.47$)$. There was no significant difference in the median length of hospital stay between the two disease groups $(\mathrm{p}=0.16)$.

\section{Discussion:}

Th17 cells are potent inducers of tissue inflammation (29) and responsible for clearing excess pathogens not adequately addressed by Th1 or Th2 cells (28). IL-17A, the principal Th17 cytokine, enhances neutrophil recruitment to sites of sepsis thereby improving bacterial clearance (34). Both animal and human models have demonstrated that IL-17A is raised in early AP (30,31) and causes pancreatic damage by inducing acinar cell necrosis (30).

We found in this study that the secreted IL-17A levels were not raised in the second hit. This may be due to two factors. Firstly IL-10 directly suppresses IL-17 in both macrophages and T cells (35). IL-10 was significantly elevated over the entire study period in the SAP group which reduced the Th17 response. Secondly TGF- $\beta$ and IL-6 are essential for Th17 cell formation (28). Galunisertib, a TGF- $\beta$ receptor I kinase inhibitor, has demonstrated the critical, stimulatory effects of TGF- $\beta$ in pancreatitis by limiting the extent of disease in animal models (36). In vivo TGF- $\beta$ has been shown to stimulate Th17 cells formation via numerous pathways (37) and levels have been shown to be elevated in early pancreatitis (38), however levels in the second week of the disease are unknown. In our study despite having a moderate adequate IL-6 response in the SAP group, stimulation of Th17 cells may be inadequate as a result of low levels of TGF- $\beta$.

The Th1 and Th2 results of this study demonstrated an immune imbalance confirming the hypothesis that an imbalance exists between SIRS and CARS in SAP patients leading to potential septic complication $(39,40)$. Previous literature is deficient in relating this immune imbalance in AP directly to clinical outcomes (17), a limitation that we have address in this study. The Th1 pro-inflammatory response (IL-2, TNF- $\alpha$ and IFN- $\gamma$ ) was not elevated in either of the disease groups. These findings are congruent with phases of AP as by day 7 the initial pro-inflammatory phase driven by SIRS is believed to have ended (10,41-47). The anti-inflammatory Th2 interleukins (IL-4 and IL-10) shows contradictory results. IL-4 demonstrated no correlation with disease severity however IL-10 was significantly elevated over the entire study period in the SAP group. IL-4 primarily triggers the Th2 response; although a positive feedback loop has been described for IL- 4 and thus the further development of Th2 cells (28). The lower than expected levels of IL-4 may be due to the timing of sample collection in that the induction of the Th2 response is largely complete by day 7 of the disease. The significantly elevated IL-10 levels in the SAP group supports 
development of immune anergy. IL-6 is recognised to mostly behave as a pro-inflammatory cytokine, however it does have antiinflammatory properties $(34,48)$. It is difficult to interpret the significantly elevated level in the SAP group. With the elevated level and its dual role, IL-6 further strengthens the concept of an immune imbalance. Although we speculate that IL-6's role during the second hit in AP is most likely pro-inflammatory. No significant correlation could be detected between the development of necrosis or sepsis in the measured cytokines.

In this study we determine the concentrations of secreted cytokines within plasma. The majority of publications reporting on cytokines in AP have used this technique. It is recognised that alternative techniques, such as intra-cellular cytokines measurements, do exist and add a different dimension. However, it has been demonstrated that the correlation between intracellular and extra-cellular cytokine concentrations determined using flow cytometry is good (49).

Increased intestinal permeability and bacterial translocation that occurs in AP is thought to be fundamental to the formation of sepsis in pancreatic necrosis. Intestinal permeability has been shown to be is altered early in SAP (50) which is further compromised by impaired splanchnic perfusion (51), all leading to the development of bacterial translocation (52). IL-17A has been shown to prevents bacterial translocation from the gut (53), however its specific role in the second hit of AP is undetermined. Gastrointestinal biopsies were not performed in this study and should be investigated in future studies.

IL-17A levels were initially evaluated by means of flow cytometry, using the Th1/Th2/Th17 CBA assay (BD Biosciences, La Jolla, Ca. USA). The mean levels for controls as well as patient samples were below the manufacturer's minimum detection limit of $18 \mathrm{pg} / \mathrm{ml}$. Hence, a confirmatory ELISA using a BioLegend Human IL-17A Max Deluxe Set was performed. This kit has a documented minimum detection value of $2 \mathrm{pg} / \mathrm{ml}$. The ELISA test confirmed the CBA flow cytometry based results.

The APACHE II and SOFA scores within this cohort of patients correlates well with disease severity. The scores were performed on the day of study enrolment, which in the majority of patients was within the first few days of illness. Sharma et al. documented an APACHE II score of greater than 14 could predict mortality in SAP with a sensitivity of $81 \%$ and specificity of $77 \%$ (8). Similarly, a mean SOFA score of 7 was determined to predict mortality in SAP (sensitivity $88 \%$ and specificity $86 \%$ ) (54). The mean APACHE II and SOFA scores in our SAP group was 17.7 and 8.2 respectively and thus mortality predictions based on these scoring systems could be applied. 
Death from late septic complications are potentially avoidable with appropriate identification and management (55). WCC, CRP and PCT are the current inflammatory markers used to identify sepsis, however differentiating inflammation and sepsis is difficult based on these markers alone (56). In our study, all these routine inflammatory markers where significantly elevated in the SAP group compared to the mild/moderate group. Interestingly, the CRP level decreased significantly over the study period in the mild/moderate group, however not so in the SAP group. The PCT level in the mild/moderate group remained normal throughout the study period, however the level in the SAP group decreased significantly.

Evaluating these routine inflammatory markers in relation to the development of necrosis and sepsis is critically important in managing the second hit. In our results, WCC proved to be the least sensitive with erratically elevated values. CRP was raised early in patients that developed necrosis but later in the patients that developed sepsis. PCT proved to be the most sensitive of the markers as it was consistently elevated in both the patients that developed necrosis as well as sepsis. This suggests that in SAP PCT is a more accurate measure of necrosis and sepsis. Serial albumin levels in the SAP group were significantly less than those in the mild/moderate group, a finding consistent with the literature (57).

Eighty percent of the patients in the severe group had local complication whereas only $27 \%$ in the mild/moderate group (all restricted to the moderate group). Assessing the moderately severe pancreatitis group in isolation, $70 \%$ of these patients developed local complications of which sterile necrosis occurred in $30 \%$ of these patients. The remaining patients had resolving organ dysfunction. Both patients in the SAP group that did not develop a local complication recovered fully with no documented evidence of sepsis. Necrosis developed in $50 \%$ of the SAP patients, of which $75 \%$ developed sepsis, confirming the association of necrosis in relation to the development of sepsis.

Sepsis was defined as either a positive blood culture or retroperitoneal air associated with a collection requiring drainage. Our unit does not perform fine needle aspirations of retroperitoneal collections to determine sepsis as we believe it increases the risk of developing sepsis. Four patients, all within the SAP group, were septic. Two patients because of systemic sepsis evidenced by positive blood cultures and two because of septic retroperitoneal collections. All SAP patients, baring one, received antibiotics. Antibiotics were most often initiated empirically by the treating surgeon/intensivist based on elevated septic markers and/or a deterioration in organ dysfunction. The use of empiric antibiotics in patients that were not proven to be septic is a confounding variable that may have influenced both the inflammatory and septic responses in these patients. The SAP patient who did not received antibiotics, died on day 8 of illness as a result of multi-organ failure. This patient had no evidence of sepsis, hence antibiotics were withheld. 
Four patients with SAP died. Two of which occurred within the first week, one in the second week and the remaining patient after 32 days of hospitalisation. One patient in the mild/moderate group died as a result of severe sepsis. An iatrogenic kidney injury occurred after a percutaneous drain was placed for a symptomatic acute fluid collection. Initially, however, the collection was sterile.

There was no significant difference in the median length of hospital stay between the two disease groups $(p=0.16)$. This finding was unexpected; however, we believe this is because of three reasons. Firstly, the mild and moderate groups were combined for statistical analysis, thus the length of hospitalization of 12 days in this group is not a true reflection of mild AP. Secondly, it is unit protocol to perform definitive surgical therapy prior to discharge (i.e. laparoscopic cholecystectomy in the case of biliary pancreatitis). Delay in definitive treatment because of long elective waiting lists, falsely increased the length of hospitalisation in several patients within the mild/moderate group. Thirdly, three patients died early in the SAP group, skewing the length of hospitalisation within this group.

A limitation of the study is that it is underpowered. However, it is powered sufficiently to detect large but not small effects sizes. For small effect sizes to be observed, a larger sample size of 82 patients is required. A further limitation is that antibiotics were instituted empirically and their usage may have confounded the development of inflammation and sepsis in certain patients. However, in severely ill patients such as SAP, antibiotics therapy without proven sepsis, could not ethically be withheld. In six patients, blood samples could not be obtained on all the required days as a result of either death, refusal of further hospitalisation and/or discharge with no further follow-up. IL-17A levels have been linked to obesity (55). Considering that body mass indexes were not recorded, an association between IL-17A, obesity and SAP could not be ascertained.

Our study confirms an immune imbalance exists in patients with SAP which may result in the development of necrosis and/or sepsis. The Th17 response, measured by IL-17A, is not active during the second hit of AP. PCT is a more sensitive marker of both necrosis and sepsis in AP. Further studies are required to evaluate the role of TGF- $\beta$ in the differentiation of the Th17 response in AP. Furthermore, additional Th17 IL's, such as IL-17F, IL-21 and IL-22, need to be evaluated to completely exclude the Th17 response during the second hit of AP.

\section{Conclusion:}

An immune imbalance exists in patients with SAP, however IL-17A is not responsible for the second hit in AP. 


\section{Acknowledgements:}

The authors thanks Dr P. Gaylard, Ms H. Tau and Dr M. Nel for their contributions.

This work was supported by grants from SAGES (Abbott Research Award), Discovery Foundation, Wits Donald Gordon Medical Centre and The University of the Witwatersrand Faculty of Health Sciences Individual Research

Grant 001283844110151211055142 and Seed Funding Grant 0012518441101512110500000000000000004550.

\section{Conflict of Interest:}

The authors declare no conflict of interest.

\section{Author Contributions:}

Data collection: JT, PF; Data analysis and interpretation: JT, MB, PF; Conception or design of the work: JT, MB, PF; Drafting the article: JT; Critical revision of the article: MB, PF; Final approval of the version to be published: JT, MB, PF. 


\section{References:}

1. Swaroop VS, Chari ST, Clain JE. Severe Acute Pancreatitis. J Am Med Assocation. 2004;291(23):2865-8.

2. Russo MW, Wei JT, Thiny MT, Gangarosa LM, Brown A, Ringel Y, et al. Digestive and liver diseases statistics, 2004. Gastroenterology. 2004 May;126(5):1448-53.

3. Lowenfels A, Sullivan T, Fiorianti J, Maisonneuve P. The epidemiology and impact of pancreatic diseases in the United States. Curr Gastroenterol Rep. 2005;7(2):90-5.

4. WHO. Global status report on noncommunicable diseases 2014. World Heal Organ. 2014;1-176.

5. Banks PA, Freeman ML. Practice guidelines in acute pancreatitis. Am J Gastroenterol. 2006 Oct;101(10):2379-400.

6. Triester SL, Kowdley K V. Prognostic factors in acute pancreatitis. J Clin Gastroenterol. 2002 Feb;34(2):167-76.

7. Dervenis C, Johnson CD, Bassi C, Bradley E, Imrie CW, McMahon MJ, et al. Diagnosis, objective assessment of severity, and management of acute pancreatitis. Santorini consensus conference. Int J Pancreatol Off J Int Assoc Pancreatol. 1999 Jun;25(3):195-210.

8. Sharma M, Banerjee D, Garg PK. Characterization of newer subgroups of fulminant and subfulminant pancreatitis associated with a high early mortality. Am J Gastroenterol. 2007;102(12):2688-95.

9. Mitchell RMS, Byrne MF, Baillie J. Pancreatitis. Lancet. 2003 Apr;361(9367):1447-55.

10. Mofidi R, Duff MD, Wigmore SJ, Madhavan KK, Garden OJ, Parks RW. Association between early systemic inflammatory response, severity of multiorgan dysfunction and death in acute pancreatitis. Br J Surg. 2006 Jun;93(6):73844.

11. Abu-Zidan FM, Bonham MJ, Windsor JA. Severity of acute pancreatitis: a multivariate analysis of oxidative stress markers and modified Glasgow criteria. Br J Surg. 2000 Aug;87(8):1019-23.

12. Neoptolemos JP, Raraty M, Finch M, Sutton R. Acute pancreatitis: the substantial human and financial costs. Gut. 1998 Jun;42(6):886-91.

13. Bhatia M, Brady M, Shokuhi S, Christmas S, Neoptolemos JP, Slavin J. Inflammatory mediators in acute pancreatitis. J Pathol. 2000 Feb;190(2):117-25.

14. Forsmark CE, Baillie J. AGA Institute Technical Review on Acute Pancreatitis. Gastroenterology. 2007;132(5):2022-44.

15. Beger HG, Büchler M. Acute Pancreatitis: Research and Clinical Management. Springer Science \& Business Media; 2012. $390 \mathrm{p}$.

16. Norman J. The role of cytokines in the pathogenesis of acute pancreatitis. Am J Surg. 1998 Jan;175(1):76-83.

17. Dambrauskas Z, Giese N, Gulbinas A, Giese T, Berberat PO, Pundzius J, et al. Different profiles of cytokine expression during mild and severe acute pancreatitis. World J Gastroenterol. 2010 Apr;16(15):1845-53.

18. Zhang J, Niu J, Yang J. Interleukin-6, interleukin-8 and interleukin-10 in estimating the severity of acute pancreatitis: an updated meta-analysis. Hepatogastroenterology. 2014 Feb;61(129):215-20.

19. Fisher CJ, Slotman GJ, Opal SM, Pribble JP, Bone RC, Emmanuel G, et al. Initial evaluation of human recombinant interleukin-1 receptor antagonist in the treatment of sepsis syndrome: a randomized, open-label, placebo-controlled multicenter trial. Crit Care Med. 1994 Jan;22(1):12-21.

20. Dinarello CA, Gelfand JA, Wolff SM. Anticytokine strategies in the treatment of the systemic inflammatory response syndrome. JAMA. 1993 Apr;269(14):1829-35.

21. Vasseur P, Devaure I, Sellier J, Delwail A, Chagneau-Derrode C, Charier F, et al. High plasma levels of the proinflammatory cytokine IL-22 and the anti-inflammatory cytokines IL-10 and IL-1ra in acute pancreatitis. Pancreatology. Jan;14(6):465-9.

22. Koussoulas V, Tzivras M, Karagianni V, Spyridaki E, Plachouras D, Giamarellou H, et al. Monocytes in systematic inflammatory response syndrome: differences between sepsis and acute pancreatitis. World J Gastroenterol WJG. 2006 Nov;12(41):6711-4.

23. Pezzilli R, Billi P, Gullo L, Beltrandi E, Maldini M, Mancini R, et al. Behavior of serum soluble interleukin-2 receptor, soluble CD8 and soluble CD4 in the early phases of acute pancreatitis. Digestion. 1994;55(4):268-73.

24. Mayer J, Rau B, Gansauge F, Beger HG. Inflammatory mediators in human acute pancreatitis: clinical and pathophysiological implications. Gut. 2000 Oct;47(4):546-52. 
25. Makhija R, Kingsnorth AN. Cytokine storm in acute pancreatitis. J Hepatobiliary Pancreat Surg. 2002;9(4):401-10.

26. Pezzilli R, Billi P, Miniero R, Barakat B. Serum interleukin-10 in human acute pancreatitis. Dig Dis Sci. 1997 Jul;42(7):1469-72.

27. Boomer JS, Green JM, Hotchkiss RS. The changing immune system in sepsis: is individualized immuno-modulatory therapy the answer? Virulence. 2014;5(1):45-56.

28. Korn T, Bettelli E, Oukka M, Kuchroo VK. IL-17 and Th17 Cells. Annu Rev Immunol. 2009;27:485-517.

29. Zambrano-Zaragoza J, Romo-Martínez E, Durán-Avelar M, García-Magallanes N, Vibanco-Pérez N. Th17 cells in autoimmune and infectious diseases. Int J Inflam. 2014;2014(II):12.

30. Ni J, Hu G, Xiong J, Shen J, Shen J, Yang L, et al. Involvement of interleukin-17A in pancreatic damage in rat experimental acute necrotizing pancreatitis. Inflammation. 2013 Feb;36(1):53-65.

31. Kay PS, Smith MD, Brand M. The Initiating Immune Response of Acute Pancreatitis May be Mediated by the T-Helper 17 Pathway. J Pancreas. 2017;18(1):241-5.

32. Banks PA, Bollen TL, Dervenis C, Gooszen HG, Johnson CD, Sarr MG, et al. Classification of acute pancreatitis--2012: revision of the Atlanta classification and definitions by international consensus. Gut. 2013 Jan;62(1):102-11.

33. Vege SS, Gardner TB, Chari ST, Munukuti P, Pearson RK, Clain JE, et al. Low mortality and high morbidity in severe acute pancreatitis without organ failure: a case for revising the Atlanta classification to include "moderately severe acute pancreatitis". Am J Gastroenterol. 2009;104(3):710-5.

34. Burkovskiy I, Sardinha J, Zhou J, Lehmann C. Cytokine release in sepsis. Adv Biosci Biotech. 2013;2013(4):860-5.

35. Gu Y, Yang J, Ouyang X, Liu W, Li H, Yang J, et al. Interleukin 10 suppresses Th17 cytokines secreted by macrophages and T cells. Eur J Immunol. 2008;38(7):1807-13.

36. Liu X, Yu M, Chen Y, Zhang J. Galunisertib (LY2157299), a transforming growth factor-\&amp;\#9 receptor I kinase inhibitor, attenuates acute pancreatitis in rats. Brazilian J Med Biol Res. 2016;49(9):1-8.

37. Qin H, Wang L, Feng T, Elson CO, Niyongere SA, Lee SJ, et al. TGF- $\beta$ promotes Th17 cell development through inhibition of SOCS3. J Immunol. 2009;183(1):97-105.

38. Jamdar S, Al-Mowallad AF, Kumar S, Siriwardena AK. Differential kinetics of plasma CD105 and transforming growth factor beta expression early in human acute pancreatitis. Pancreas. 2006;32(2):152-8.

39. Bone RC. Sir Isaac Newton, sepsis, SIRS, and CARS. Crit Care Med. 1996 Jul;24(7):1125-8.

40. Kox WJ, Volk T, Kox SN, Volk HD. Immunomodulatory therapies in sepsis. Intensive Care Med. 2000;26 Suppl 1:S124128.

41. McKay CJ, Imrie CW. The continuing challenge of early mortality in acute pancreatitis. Br J Surg. 2004 Oct;91(10):1243-4.

42. Werner J, Feuerbach S, Uhl W, Büchler MW. Management of acute pancreatitis: from surgery to interventional intensive care. Gut. 2005 Mar;54(3):426-36.

43. Singh VK, Wu BU, Bollen TL, Repas K, Maurer R, Mortele KJ, et al. Early systemic inflammatory response syndrome is associated with severe acute pancreatitis. Clin Gastroenterol Hepatol Off Clin Pract J Am Gastroenterol Assoc. 2009 Nov;7(11):1247-51.

44. Renner IG, Savage WT, Pantoja JL, Renner VJ. Death due to acute pancreatitis. A retrospective analysis of 405 autopsy cases. Dig Dis Sci. 1985 Oct;30(10):1005-18.

45. Johnson CD, Abu-Hilal M. Persistent organ failure during the first week as a marker of fatal outcome in acute pancreatitis. Gut. 2004 Sep;53(9):1340-4.

46. UK guidelines for the management of acute pancreatitis. Gut. 2005 May;54 Suppl 3:iii1-9.

47. Buter A, Imrie CW, Carter CR, Evans S, McKay CJ. Dynamic nature of early organ dysfunction determines outcome in acute pancreatitis. Br J Surg. 2002 Mar;89(3):298-302.

48. Scheller J, Chalaris A, Schmidt-Arras D, Rose-John S. The pro- and anti-inflammatory properties of the cytokine interleukin-6. Biochim Biophys Acta - Mol Cell Res. 2011;1813(5):878-88.

49. Schuerwegh a J. Comparison of intracellular cytokine production with extracellular cytokine levels using two flow cytometric techniques. Cytometry B Clin Cytom. 2003;55(1):52-8. 
50. Rahman SH, Ammori BJ, Holmfield J, Larvin M, McMahon MJ, Sarr MG, et al. Intestinal hypoperfusion contributes to gut barrier failure in severe acute pancreatitis. J Gastrointest Surg. 2003;7(1):26-36.

51. Inoue K, Hirota M, Kimura Y, Kuwata K, Ohmuraya M, Ogawa M. Further evidence for endothelin as an important mediator of pancreatic and intestinal ischemia in severe acute pancreatitis. Pancreas. 2003 Apr;26(3):218-23.

52. Bakker OJ, van Santvoort HC, Besselink MGH, van der Harst E, Hofker HS, Gooszen HG. Prevention, detection, and management of infected necrosis in severe acute pancreatitis. Curr Gastroenterol Rep. 2009;11:104-10.

53. Blaschitz C, Raffatellu M. Th17 cytokines and the gut mucosal barrier. J Clin Immunol. 2010;30(2):196-203.

54. Adam F, Bor C, Uyar M, Demirağ K, Çankayali I. Severe acute pancreatitis admitted to intensive care unit: SOFA is superior to Ranson's criteria and APACHE II in determining prognosis. Turkish J Gastroenterol. 2013;24(5):430-5.

55. Lowham A, Lavelle J, Leese T. Mortality from Acute Pancreatitis. Int J Pancreatol. 1999;25(2):103-6.

56. Castelli GP, Pognani C, Meisner M, Stuani A, Bellomi D, Sgarbi L. Procalcitonin and C-reactive protein during systemic inflammatory response syndrome, sepsis and organ dysfunction. Crit Care. 2004;8(4):R234-42.

57. Li S, Zhang Y, Li M, Xie C, Wu H. Serum albumin, a good indicator of persistent organ failure in acute pancreatitis. BMC Gastroenterol. 2017;17(1):59. 